\title{
Resonance Raman enhancement for photoinduced polaronic states of a quasi-one-dimensional mixed-valence platinum complex
}

\author{
J. Takeda, M. Okada, and S. Kurita \\ Laboratory of Applied Physics, Faculty of Engineering, Yokohama National University, Yokohama 240, Japan \\ K. Tanaka and T. Suemoto \\ Institute for Solid State Physics, University of Tokyo, Minato-ku 106, Tokyo, Japan
}

(Received 5 July 1995)

\begin{abstract}
Clear resonance enhancement curves of several Raman modes associated with the photoinduced defect states of the quasi-one-dimensional mixed-valence complex $\left[\mathrm{Pt}(\mathrm{en})_{2}\right]\left[\mathrm{Pt}(\mathrm{en})_{2} \mathrm{Cl}_{2}\right]\left(\mathrm{ClO}_{4}\right)_{4}$ were observed in the wide energy region covering the photoinduced absorption bands. They are classified into two groups with different resonant energies. This separation of the resonant energies indicates very strongly the photoinduced defect states of this material being polarons rather than neutral solitons and the asymmetry between the hole and the electron polarons.
\end{abstract}

Since the pioneering work on polyacetylene, ${ }^{1}$ nonlinear excitations (neutral and charged solitons, polarons, and bipolarons) associated with the lattice instability in onedimensional (1D) half-filled electron systems have been attracting much attention in recent years. Halogen bridged metal complexes (the $M X$ chain complexes), which comprise linear chains $\left(-M^{2+}-X^{-}\right.$$\left.M^{4+}-X^{-}-M^{2+}-X^{-}-M^{4+}-X^{-}-\right) \quad(M=\mathrm{Pt}, \mathrm{Pd}, \mathrm{Ni} \quad$ and $X=\mathrm{Cl}, \mathrm{Br}, \mathrm{I})$, are the best materials to study such nonlinear excitations for the following reasons. First, these materials are the $1 \mathrm{D}$ systems not only in the crystal structure but also in the electronic configuration, ${ }^{2-4}$ Second, the good quality samples are obtained in comparison with the $1 \mathrm{D}$ organic compounds such as polyacetylene. Third, the ground state of the $M X$ chain complexes has a twofold degeneracy, which allows the solitonlike excitations, and is characterized as a $1 \mathrm{D}$ charge density wave (CDW) state arising from a charge disproportion on the $M$ sublattice sites accompanied with substantial distortion of the $X$ sublattice. Finally, the physical parameters such as the displacement of the bridging halogen and the dimensionality of the CDW can be easily controlled by changing the $M$ and the $X$ atoms, the legend molecule, and the counteranion surrounding them. ${ }^{5,6}$

$\left[\mathrm{Pt}(\mathrm{en})_{2}\right]\left[\mathrm{Pt}(\mathrm{en})_{2} \mathrm{Cl}_{2}\right]\left(\mathrm{ClO}_{4}\right)_{4} \quad$ (where en denotes ethylenediamine), hereafter referred to as $\mathrm{Pt}-\mathrm{Cl}$, is one of the typical $M X$ chain complexes, which clearly shows the photoinduced absorption bands due to the nonlinear excitations in the intragap region below the intervalence charge-transfer (CT) absorption band. ${ }^{7}$ The intensity of the electron spin resonance increases in the same rate as that of the photoinduced absorption bands by the light irradiation. ${ }^{8-10}$ Therefore the origin of the photoinduced defect state in $\mathrm{Pt}-\mathrm{Cl}$ is considered to be the neutral solitons or the polarons. However, it is still controversial that either the neutral soliton or the polaron is the relevant defect states, in spite of the large amount of the experimental and theoretical studies about the photoinduced defect states of $\mathrm{Pt}-\mathrm{Cl}^{7-17}$
Donohoe and co-workers found several Raman modes associated with the photoinduced defect states in $\mathrm{Pt}-\mathrm{Cl}$ and pointed out that the origin of these modes is due to the hole and the electron polarons. ${ }^{18,19}$ However, they did not obtain the resonance enhancement curves of these Raman modes, which are crucial to the identification of the origin. In this paper, we report the clear resonance enhancement curves of the Raman modes in the wide energy region covering the photoinduced absorption bands ( $A$ and $B$ bands) in $\mathrm{Pt}-\mathrm{Cl}$, which follows our recent preliminary report. ${ }^{20}$ The origin of these modes is attributed to the hole and the electron polarons by comparing the observed results with the calculation of the two-band theory. ${ }^{21,22}$

The single crystal of $\mathrm{Pt}-\mathrm{Cl}$ were synthesized by the procedure previously described. ${ }^{4}$ The samples were mounted on a copper block and kept at $16 \mathrm{~K}$ in a temperaturecontrolled cryostat. The photolysis of the samples was performed for $150 \mathrm{~min}$ by the $365-\mathrm{nm}$ line from a mercury lamp with a power of $1 \mathrm{~mW} / \mathrm{cm}^{2}$. The photoinduced defects were efficiently produced inside the $\mathrm{Pt}-\mathrm{Cl}$ crystals by the photolysis since the quantum efficiency of the generation of the photoinduced defects is large for the excitation by the uv light. ${ }^{7}$

The light source of the resonance Raman spectroscopy was provided by a Ti:sapphire laser, DCM (4-dicyanomethylene-2-methyl-6-P-dimethylaminostyryl4H-pyran) and rhodamine $6 \mathrm{G}$ dye lasers pumped by an Ar-ion laser, and a He-Ne laser. The excitation photon energy was varied from 1.44 to $2.20 \mathrm{eV}$, corresponding to the whole energy region of the $A$ and the $B$ bands. The incident light impinged near normally upon the sample's surface and the scattered light was measured in the backward-scattering configuration. The scattered light was analyzed by a triple monochromator and a charge coupled device detector cooled by liquid nitrogen. The polarization of the incident and scattered light was parallel to the $b$ axis of the sample (E\|b).

Figure 1 shows the resonance Raman spectra of $\mathrm{Pt}-\mathrm{Cl}$ 


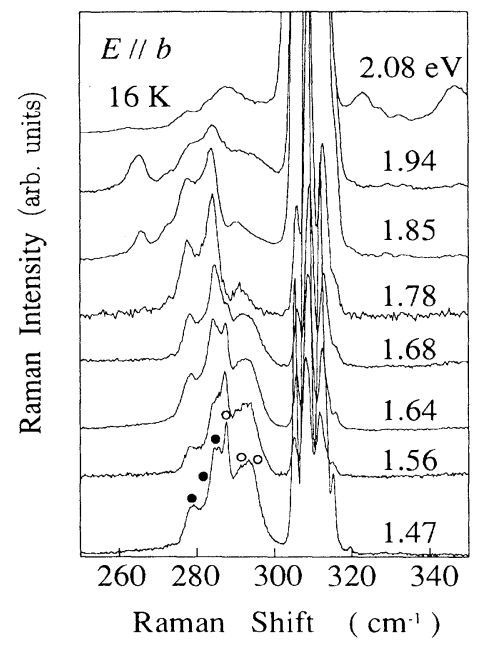

FIG. 1. Resonance Raman spectra of $\mathrm{Pt}-\mathrm{Cl}$ with different incident photon energies in the $A$ and the $B$ band regions after the photolysis.

with different incident photon energies in the $A$ and the $B$ band regions. Below the symmetric stretching mode $v_{1}$ $\left(\sim 308 \mathrm{~cm}^{-1}\right)$ of $\mathrm{Pt}-\mathrm{Cl}$, we found six Raman lines (as shown by open and closed circles) for all of the incident photon energies and one additional Raman line (264.1 $\mathrm{cm}^{-1}$ ) for those of the $B$ band region. The six Raman lines were obtained from the deconvolution of the spectral structures between 270 and $300 \mathrm{~cm}^{-1}$ as mentioned below. As seen in Fig. 1, the intensity ratios between the higher-frequency modes (open circles) and the lowerfrequency modes (closed circles) depend on the incident photon energy, strongly implying that the resonant energy of the higher-frequency modes is different from that of the lower ones.

Figure 2 shows the observed resonance Raman spectrum and the analytical ones obtained from the deconvolution procedure for the incident photon energy $1.64 \mathrm{eV}$. The observed Raman spectrum was deconvoluted into six

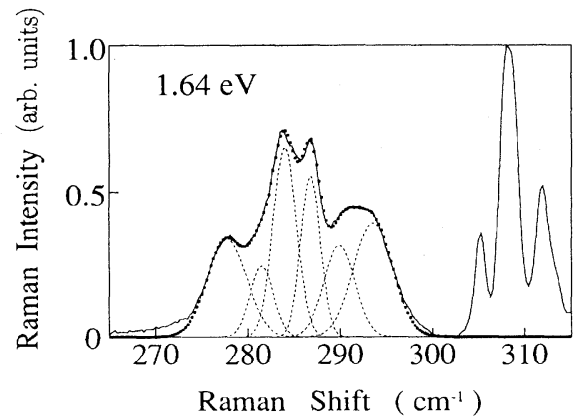

FIG. 2. Typical observed resonance Raman spectrum (solid curve), the six Raman components obtained from the deconvolution of the observed Raman spectrum (broken curves), and the spectrum given by the superposition of the six Raman components (dotted curve).
Gaussian components by the method of a least-squares fit. The broken curves show the six Raman components obtained from the deconvolution. The dotted curve indicates the superposition of the six components and is in quite good agreement with the observed Raman spectrum between 270 and $300 \mathrm{~cm}^{-1}$. The peak position and the half-width of the six Raman components are listed in Table I. The analytical errors of the peak position and the half-width are also indicated in parentheses. The half-width of the 281.6-, 284.3-, and $286.9-\mathrm{cm}^{-1}$ lines is about $3.0 \mathrm{~cm}^{-1}$, being equivalent to that of the experimental spectral resolution. On the other hand, the half width of the 278.0-, 289.9-, 293.9-, and 264.1- $\mathrm{cm}^{-1}$ lines is about $4-6 \mathrm{~cm}^{-1}$, implying complexities of these lines. However, since the peak position and the half-width of these six lines do not change with the incident photon energy and the additional Raman modes were not observed between 270 and $300 \mathrm{~cm}^{-1}$, we assume that the spectral structure between 270 and $300 \mathrm{~cm}^{-1}$ consists of the six Raman modes.

The absorption coefficient of the incident light becomes quite large after the photolysis, ${ }^{7,20}$ implying that the penetration depth of the incident light is within the thickness in which the photoinduced defects exist. On the other hand, the absorption coefficient and the reflectivity of the scattered light are nearly constant in the energy region considered, since the photoinduced absorption and the reflectance spectra are very broad in $\mathrm{Pt}-\mathrm{Cl}$. Thus the corrected intensity due to the penetration depth of the incident light and the reabsorption of the scattered light for the $270-300-\mathrm{cm}^{-1}$ Raman lines can be obtained by normalizing the intensity of these Raman lines by that of the $v_{1}$ mode. The corrected Raman cross section of the $270-300-\mathrm{cm}^{-1}$ modes $\sigma_{d}(E)$ is given by $\left\{I_{d}(E) /\right.$ $\left.I_{s}(E)\right\} \sigma_{s}(E)$, where $I_{d}(E)$ and $I_{s}(E)$ denote the observed Raman intensities of the 270-300- $\mathrm{cm}^{-1}$ modes and the $v_{1}$ mode, respectively, and $\sigma_{s}(E)$ denotes the Raman cross section of the $v_{1}$ mode. ${ }^{23}$

The Raman cross section $\sigma_{d}(E)$ of the $270-300-\mathrm{cm}^{-1}$ modes as a function of incident photon energy is shown in Figs. 3(a) and 3(b) in a logarithmic scale. The typical photoinduced absorption bands of $\mathrm{Pt}-\mathrm{Cl}$ is also shown on a linear scale. The analytical error for the intensity of the Raman cross section, coming from the deconvolution procedure, is within the marks shown by the circles and triangles. The higher-frequency Raman modes [Fig. 3(a)] show a strong resonance enhancement at the lower-

TABLE I. Peak position and half-width of the six Raman components obtained from the deconvolution procedure.

\begin{tabular}{ll}
\hline $\begin{array}{c}\text { Peak } \\
\text { position }\left(\mathrm{cm}^{-1}\right)\end{array}$ & Half-width $\left(\mathrm{cm}^{-1}\right)$ \\
\hline $278.0( \pm .0 .2)$ & $5.1( \pm 0.2)$ \\
$281.6( \pm .0 .3)$ & $3.2( \pm .0 .3)$ \\
$284.3( \pm 0.2)$ & $3.0( \pm 0.2)$ \\
$286.9( \pm 0.2)$ & $2.7( \pm 0.2)$ \\
$289.9( \pm 0.3)$ & $4.1( \pm 0.2)$ \\
$293.9( \pm 0.2)$ & $5.4( \pm 0.2)$ \\
\hline \hline
\end{tabular}




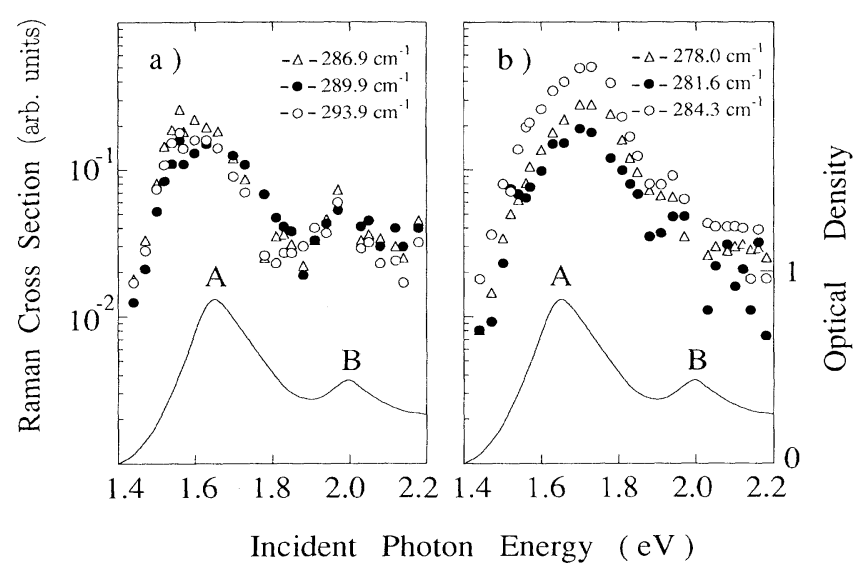

FIG. 3. Raman cross sections of the (a) higher- and (b) lower-frequency Raman modes as a function of incident photon energy in a logarithmic scale. Typical photoinduced absorption bands ( $A$ and $B$ bands) are also shown on a linear scale.

energy side of the $A$ band $(1.60 \mathrm{eV})$ and the peak energy of the $B$ band $(1.97 \mathrm{eV})$. On the other hand, the lowerfrequency Raman modes [Fig. 3(b)] show a strong resonance enhancement at the higher-energy side of the $A$ band $(1.72 \mathrm{eV})$, but no clear enhancement in the energy region of the $B$ band. The separation of the resonant energy in the $A$ band region for the two classes of Raman modes strongly indicates that the $270-300-\mathrm{cm}^{-1}$ Raman modes are associated with two kinds of the photoinduced defect states in Pt-Cl.

According to the three-quarter-filled two-band mod$\mathrm{el},{ }^{21,22}$ the asymmetry between the hole and the electron polarons exists and the energy of the electronic states of the hole polarons is a little higher $(0.1-0.2 \mathrm{eV})$ than that of the electron polarons in both the $A$ and the $B$ band regions in Pt-Cl. The energies of the photoinduced absorption bands obtained from the absorption spectroscopy, the resonance Raman energies obtained in this study, and the theoretical calculation are listed in Table II. The rel-

TABLE II. Energies of the photoinduced absorption bands ( $A$ and $B$ bands), resonance Raman energies, and calculated energies of the polaronic states of $\mathrm{Pt}-\mathrm{Cl}$. Notations $\boldsymbol{P}^{-}$and $\boldsymbol{P}^{+}$ denote the electron and the hole polarons, respectively. The unit of the energy is $\mathrm{eV}$.

\begin{tabular}{lcc}
\hline \hline & $A$ band & $B$ band \\
\hline $\begin{array}{l}\text { Expt. } \\
\text { absorption measurement }\end{array}$ & $1.68(0.70)$ & $1.98(0.83)$ \\
$\begin{array}{l}\text { Raman measurement } \\
\text { b }\end{array}$ & & \\
$\quad 286.9-293.9 \mathrm{~cm}^{-1}$ & $1.60(0.65)$ & $1.97(0.83)$ \\
$278.0-284.3 \mathrm{~cm}^{-1}$ & $1.72(0.72)$ & \\
& & \\
Theory $^{\mathrm{c}}$ & & \\
$P^{-}$ & $1.44(0.60)$ & $2.06(0.86)$ \\
$P^{+}$ & $1.54(0.64)$ & $2.26(0.94)$ \\
\hline \hline
\end{tabular}

${ }^{\mathrm{a}}$ Reference 7.

${ }^{\mathrm{b}}$ Present study.

${ }^{\mathrm{c}}$ Reference 22 . ative energies of the photoinduced absorption bands to the energy of the CT absorption band are also presented in parentheses.

A comparison of the results shown in Table II strongly implies that the lower-frequency Raman modes and the higher-frequency ones are associated with the hole and the electron polarons, respectively, although the observed resonant energies are a little higher than the calculated ones. The observed resonant energy difference between the lower- and the higher-frequency Raman modes is about $0.1 \mathrm{eV}$ in the $A$ band region, coinciding well with the energy difference between the hole and the electron polaron states obtained from the theoretical calculation.

The peak energy of the $A$ band $(1.68 \mathrm{eV})$ is located between the observed resonant energy of the higherfrequency Raman modes $(1.60 \mathrm{eV})$ and that of the lowerfrequency modes $(1.72 \mathrm{eV})$, implying that the $A$ band consists of the superposition of the photoinduced absorption bands due to the hole and the electron polarons. Because the hole and the electron polarons are equally produced in the $M X$ chains by the photolysis, we would not separately observe the two kinds of the photoinduced absorption bands in the usual absorption spectroscopy. On the other hand, the resonance Raman spectroscopy we performed is very sensitive to separately observe the different defect states, thus being able to find the asymmetry between the hole and the electron polarons.

According to Gammel et al., the calculated absorption intensity of the electron polarons in the $A$ and the $B$ bands and that of the hole polarons in the $A$ band are strong, while that of the hole polarons in the $B$ band is rather weak. ${ }^{21}$ Moreover, the resonant energy due to the hole polarons in the $B$ band region is just below the strong absorption CT band. Thus a clear resonance enhancement of the lower-frequency Raman modes due to the hole polarons may not be observed in the $B$ band region, coinciding with our results shown in Fig. 3(b).

Bulou, Donohoe, and Swanson calculated the Raman frequencies due to various kinds of the defect states in $\mathrm{Pt}-\mathrm{Cl}$ and found that the Raman frequency of the electron polarons $\left(255-285 \mathrm{~cm}^{-1}\right)$ is softer than that of the hole polarons $\left(285-300 \mathrm{~cm}^{-1}\right) \cdot{ }^{24}$ However, the calculated Raman frequencies depend on the magnitude of the parameter $K_{2} / K_{1}\left(K_{1}\right.$ and $K_{2}$ representing the $\mathrm{Pt}^{4+}-\mathrm{Cl}$ and $\mathrm{Pt}^{2+}-\mathrm{Cl}$ force constants and $K_{2} / K_{1}=0.3$ in their calculation) and even the order of the frequency between the hole and the electron polarons may be reversed if $K_{2} / K_{1}$ becomes smaller than 0.3. Therefore, our assertion that the lower-frequency Raman modes correspond to the hole polarons and that of the higher-frequency Raman modes to the electron polarons might not contradict the calculation.

According to Bulou, Donohoe, and Swanson's calculation, many Raman active modes (more than six) associated with the electron polarons, a pair of the electron polarons, and the hole polarons exist in the frequency region between 260 and $300 \mathrm{~cm}^{-1}$. The half-width of the 278.0-, 289.9-, 293.9-, and 264.1- $\mathrm{cm}^{-1}$ Raman lines we observed are broader than the experimental spectral resolution, implying that these lines may consist of superposition of a few Raman modes coming from the isotropic effect of the 
chlorine atoms.

The 264.1- $\mathrm{cm}^{-1}$ Raman mode is observed only in the light excitation near the $B$ band peak (where the resonant energy of the electron polaron locates) as shown in Fig. 1 and the relative intensity of this mode to that of the $v_{1}$ mode increases with the irradiation time of the uv light. ${ }^{25}$ Thus this mode must be also relevant to the electron polarons. Since the observed Raman intensity of the 264.1$\mathrm{cm}^{-1}$ mode seems to be weaker than that of the other modes and the reabsorption effect of the scattered light is large because of the strong absorption of the $A$ band region, this mode may not be observed in the $A$ band region.

The optical absorption bands due to the neutral solitons appear at 1.15 and $2.38 \mathrm{eV}$ in $\mathrm{Pt}-\mathrm{Cl}$ according to the calculation of the two-band model. ${ }^{22}$ These energies are quite different from the observed resonance Raman energies shown in Table II. Moreover, the separation of the resonant energy between the higher- and the lowerfrequency modes in the $A$ band region we observed is unlikely to come from the only one midgap absorption band $(1.15 \mathrm{eV})$ due to the neutral solitons.

In conclusion, we observed the clear resonance enhancement curves of several Raman modes associated with the photoinduced defect states in the wide energy region covering the $A$ and the $B$ bands in $\mathrm{Pt}-\mathrm{Cl}$. From the resonant curves of these Raman modes, the origins of these modes are attributed to the hole and the electron polarons rather than the neutral solitons by comparing the observed results with the calculation of the two-band theory. We also found that the energy of the hole polaronic states are located at $1.72 \mathrm{eV}$ for the $A$ band and those of the electron polaronic states lie at 1.60 and 1.97 $\mathrm{eV}$ for the $A$ and the $B$ band regions in $\mathrm{Pt}-\mathrm{Cl}$, respectively. These results show that the resonance Raman spectroscopy for the photoinduced defect states is a more sensitive method than the usual absorption spectroscopy in order to elucidate the origin of the nonlinear excitations.

The electron and the hole polarons are equally produced in the $M X$ chains by the photolysis. On the other hand, only hole polarons are effectively produced in the $M X$ chains by the halogen doping. ${ }^{12}$ Thus the origin of the Raman modes between 260 and $300 \mathrm{~cm}^{-1}$ might be better studied by the resonance Raman scattering for the halogen-doped samples. This experiment is now in progress.

We would like to thank Professor M. Tanaka of Yokohama National University for showing us his detailed data of the Raman cross section of the $v_{1}$ mode of Pt-Cl. One of us (J.T.) also acknowledges the financial support for this study by the foundation of Kanagawa Academy of Science and Technology.
${ }^{1}$ W. P. Su, J. R. Schrieffer, and A. J. Heeger, Phys. Rev. Lett. 42, 1698 (1979); Phys. Rev. B 22, 2099 (1980).

${ }^{2}$ N. Matsumoto, M. Yamashita, I. Ueda, and S. Kida, Mem. Fac. Sci. Kyushu Univ. Ser. C 11, 207 (1978).

${ }^{3}$ M. Tanaka, S. Kurita, T. Kojima, and Y. Yamada, Chem. Phys. 91, 257 (1984).

${ }^{4}$ M. Tanaka, S. Kurita, M. Fujisawa, and S. Matsumoto, J. Phys. Soc. Jpn. 54, 3632 (1985); M. Tanaka, W. Kaneko, S. Kurita, A. Yamada, and H. Fukutani, ibid. 56, 1197 (1987).

${ }^{5}$ Y. Wada, T. Mitani, M. Yamashita, and T. Koda, J. Phys. Soc. Jpn. 54, 3143 (1985).

${ }^{6}$ H. Okamoto, T. Mitani, K. Toriumi, and M. Yamashita, Phys. Rev. Lett. 69, 2248 (1992); H. Okamoto, Y. Oka, T. Mitani, K. Toriumi, and M. Yamashita, Mol. Cryst. Liq. Cryst. 256, 161 (1994).

${ }^{7}$ S. Kurita, M. Haruki, and K. Miyagawa, J. Phys. Soc. Jpn. 57, 1789 (1988).

${ }^{8}$ S. Kurita and M. Haruki, Synth. Metals 29, F129 (1989).

${ }^{9}$ N. Kuroda, M. Ito, Y. Nishina, A. Kawamori, Y. Kodera, and T. Matsukawa, Phy. Rev. B 48, 4245 (1993).

${ }^{10}$ C. A. Arrington, C. J. Unkefer, R. J. Donohoe, S. C. Huckett, S. Kurita, and B. I. Swanson, Solid State Commun. 84, 979 (1992).

${ }^{11}$ N. Kuroda, M. Sakai, Y. Nishina, M. Tanaka, and S. Kurita,
Phys. Rev. Lett. 58, 2122 (1987).

${ }^{12}$ M. Haruki and S. Kurita, Phys. Rev. B 39, 5706 (1989).

${ }^{13}$ R. J. Donohoe, S. A. Ekberg, C. D. Tait, and B. I. Swanson, Solid State Commun. 71, 49 (1989).

${ }^{14}$ D. Baeriswyl and A. R. Bishop, J. Phys. C 21, 339 (1988).

${ }^{15}$ A. Mishima and K. Nasu, Phys. Rev. B 39, 5758 (1989); 39, 5763 (1989); 40, 5593 (1989).

${ }^{16}$ Y. Tagawa and N. Suzuki, J. Phys. Soc. Jpn. 59, 4074 (1990).

${ }^{17}$ K. Iwano and K. Nasu, J. Phys. Soc. Jpn. 61, 1380 (1992).

${ }^{18}$ R. J. Donohoe, C. D. Tait, and B. I. Swanson, Chem. Mater. 2, 315 (1990).

${ }^{19}$ R. J. Donohoe, R. B. Dyer, and B. I. Swanson, Solid State Commun. 73, 521 (1990).

${ }^{20}$ J. Takeda et al., Mol. Cryst. Liq. Cryst. 256, 873 (1994).

${ }^{21}$ J. T. Gammel, R. J. Donohoe, A. R. Bishop, and B. I. Swanson, Phys. Rev. B 42, 10566 (1990).

${ }^{22}$ J. T Gammel, A. Saxena, I. Batistic, A. R. Bishop, and S. R. Phillpot, Phys. Rev. B 45, 6408 (1992).

${ }^{23}$ M. Tanaka, S. Kurita, Y. Okada, T. Kojima, and Y. Yamada, Chem. Phys. 96, 343 (1985); M. Tanaka (unpublished).

${ }^{24}$ A. Bulou, R. J. Donohoe, and B. I. Swanson, J. Phys. Condens. Matter 3, 1709 (1991)

25 J. Takeda and S. Kurita (unpublished). 